\title{
Papers
}

\section{Modelling cost effectiveness of meningococcal serogroup C conjugate vaccination campaign in England and Wales}

Caroline L Trotter, W John Edmunds

\begin{abstract}
Objectives To assess the cost effectiveness of a meningococcal serogroup $\mathrm{C}$ conjugate vaccination campaign in 0-17 year olds.

Design Cost effectiveness analysis from the perspective of the healthcare provider.

Setting England and Wales.

Main outcome measure Cost per life year saved.

Results In 1998-9, immediately before the introduction of meningococcal $\mathrm{C}$ vaccination, the burden of serogroup $\mathrm{C}$ disease was considerable, with an estimated 1137 cases in people aged $0-17$ years and at least 72 deaths. The vaccination campaign is estimated to have cost between $£ 126 \mathrm{~m}(\$ 180 \mathrm{~m}$, $€ 207 \mathrm{~m})$ and $£ 241 \mathrm{~m}$ (\$343m, €395m), depending on the price of the vaccine. Under base case assumptions the cost per life year saved from the vaccination campaign is estimated to be $£ 6259$. School based vaccination was more cost effective than general practice based vaccination because of lower delivery costs. Immunisation of infants aged under 1 year was the least cost effective component of the campaign because, although this maximises the life years gained, the three dose schedule required is more expensive than other methods of delivery. Estimates of the cost per life year saved were sensitive to assumptions on the future incidence of disease and the case fatality ratio.

Conclusions Meningococcal C vaccination is likely to be more cost effective in all age groups when the incidence of disease is high. It is also more cost effective when given to children aged 1-4 (by general practitioners) and to children and young people aged 5-17 years at school than when administered to infants under 12 months of age or young people aged 16-17 years who are not at school.
\end{abstract}

\section{Introduction}

In November 1999 the UK Department of Health incorporated meningococcal serogroup $\mathrm{C}$ conjugate (MenC) vaccine into routine infant immunisation and launched a national campaign offering vaccine to everyone aged under 18 years. ${ }^{1}$ The accelerated testing and introduction of this vaccine was prompted by a growing burden of meningococcal disease from the mid-1990s onwards, with serogroup C becoming more important. ${ }^{2}$ The vaccine provided a new opportunity for primary prevention of meningococcal disease because, unlike the older polysaccharide vaccines, it is immunogenic in infants and primes for memory. ${ }^{3}$

We considered the cost effectiveness of the UK vaccination campaign in terms of the cost per life year saved from the perspective of the healthcare provider (the NHS). We considered only costs and savings accruing to the NHS compared with the life years saved. We ignored private costs and private benefits, except loss of life.

\section{Methods}

We compared the cost effectiveness of the meningococcal C vaccine campaign with the previous strategy, when there was no national vaccination programme and cases were treated as they arose, with control measures implemented in the event of an outbreak. ${ }^{4}$ We compared the cost effectiveness of different components of the UK vaccination strategy (such as routine versus catch up, school based versus general practitioner based immunisation).

\section{Cohort model}

Because no clinical trial of vaccine efficacy exists we constructed a model to estimate the direct impact of the campaign by following an imaginary vaccine campaign cohort over a lifetime. This comprised 18 birth cohorts of people aged 0-17 who were offered vaccine in the first year of the campaign. We assumed there were 658800 individuals in each cohort at birth, which is the average size of birth cohorts over the past 18 years (source: Office for National Statistics). We calculated the number of cases of serogroup C meningococcal disease per year by multiplying estimated incidence of disease by the susceptible population. In all cases patients were assumed to die or to acquire lifelong immunity to meningococcal disease and so were removed from the susceptible pool. Those dying from serogroup C meningococcal disease were assumed to lose the average life expectancy for the age at which they died.

\section{Sensitivity analysis}

The base case scenario was considered to be the most likely set of parameters. However, because of uncertainty surrounding these estimates we explored a range of values. Firstly, we varied one parameter at a time within its given range in a univariate sensitivity

\section{Immunisation Division, PHLS Communicable Disease Surveillance Centre, London NW9 5EQ Caroline L Trotter research scientist \\ Department of Economics, City University, London EC1V $0 \mathrm{HB}$ W John Edmunds research fellow \\ Correspondence to: C Trotter \\ ctrotter@phls.org.uk}

BMJ 2002;324:1-6 
analysis. Secondly, we performed a multivariate sensitivity analysis, under six different scenarios of disease burden, using Monte Carlo simulation with @RISK 4.0 (Palisade Corporation, NY, USA) running within Microsoft Excel. Here, we drew input parameter values from a probability distribution using Latin hypercube sampling. Uniform distributions were assumed for all input parameter values in the base case. The model was simulated 500 times to generate a distribution of outcome values.

\section{Costs and discounting}

We measured all costs in pounds sterling at 2000 prices, with costs estimated from previous years inflated using the hospital and community health services pay and prices index. Future costs and benefits were discounted back to their present value, with the assumption that all costs and benefits occurred at the end of the year. In the base case we used a 3\% discount rate for both costs and benefits, as recommended by the US panel. ${ }^{5}$ We investigated a range of other discount rates because national recommendations differ-for example, the UK Department of Health recommends discount rates of $1.5 \%$ for benefits and $6 \%$ for costs. ${ }^{6}$

\section{Estimating model parameters}

In the base case we derived future incidence of serogroup C disease from 1998-9 estimates because this was the period immediately before the introduction of the vaccine. The incidence of notified and laboratory confirmed meningococcal disease in the United Kingdom had increased every year from 1995, and the proportion of cases attributable to serogroup C increased from $25 \%$ to almost $40 \%{ }^{7}$ because of the introduction and spread of a virulent clone (C2a, electrophoretic type 15) over this time period. To explore the effects of a lower incidence in the sensitivity analysis we also estimated the average incidence of serogroup C disease between 1989-90 and 1994-5 (table 1).

We obtained data on serogroup and age from 1989-99 from the meningococcal reference unit of the Public Health Laboratory Service. Not all infections are confirmed by a laboratory, and not all confirmed cases are characterised by serogroup, ${ }^{8}$ so we adjusted these data to estimate the true incidence of disease. Firstly, we estimated underascertainment by comparing reference unit data with hospital episode statistics and derived scale up factors. The hospital episode statistics (www.doh.gov.uk/hes) contain details of all

Table 1 Estimates of annual incidence of meningococcal group $C$ disease and case fatality ratios in England and Wales

\begin{tabular}{|c|c|c|c|c|c|}
\hline \multirow{2}{*}{$\begin{array}{l}\text { Age group } \\
\text { (years) }\end{array}$} & \multicolumn{2}{|c|}{ Disease incidence/100 000} & \multicolumn{3}{|c|}{ Case fatality ratio (\%) } \\
\hline & High (1998-9) & Low (1989-94) & Low & Medium & High \\
\hline$<1$ & 31.53 & 9.64 & 4.3 & 6.2 & 8.6 \\
\hline $1-4$ & 16.09 & 4.57 & 4.7 & 7.1 & 9.5 \\
\hline $5-9$ & 5.77 & 1.27 & 1.6 & 2.8 & 3.4 \\
\hline$\overline{10-14}$ & 4.97 & 1.02 & 5.5 & 6.6 & 10.1 \\
\hline 15-19 & 7.92 & 2.15 & 14.3 & 16.5 & 17.9 \\
\hline $20-24$ & 1.89 & 0.71 & 8.1 & 8.0 & 11.4 \\
\hline $25-44$ & 0.67 & 0.23 & 12.6 & 12.8 & 14.5 \\
\hline $45-64$ & 0.77 & 0.25 & 13.6 & 16.4 & 16.0 \\
\hline$\geqslant 65$ & 0.50 & 0.23 & 31.6 & 36.0 & 30.6 \\
\hline Overall & 2.94 & 0.84 & 7.8 & 9.6 & 12.6 \\
\hline
\end{tabular}

patients admitted to and treated in NHS hospitals in England and is likely to be the most complete source of information on meningococcal disease. Cases were classified as meningococcal disease if there were ICD-10 (international classification of diseases, 10th revision) codes A39.0-A39.9 in any of the seven diagnostic fields (ICD-9 codes used before 1996). Secondly, after 1996 (when diagnosis with the polymerase chain reaction was widely introduced) cases confirmed by polymerase chain reaction but uncharacterised were proportionally redistributed among other serogroups.

Deaths from meningococcal disease are registered with the Office for National Statistics with ICD-9 disease specific codes; and these are routinely linked to reference unit laboratory reports. In 1998-9 there were 72 registered deaths from laboratory confirmed serogroup C disease in $0-17$ year olds. This is a minimum estimate as not all cases are confirmed by a laboratory and not all deaths will be registered to codes specific to meningococcal disease. Because of this uncertainty we used a range of mortality estimates in the sensitivity analysis. For the high estimate we calculated age specific case fatality ratios by dividing the number of laboratory confirmed registered deaths by the number of laboratory confirmed cases in 1998-9. For the low estimate we calculated the case fatality ratio by dividing the actual number of laboratory confirmed registered deaths by the adjusted number of cases for 1998-9 (described above). The medium estimate, used in the base case, was the mean of the high and low estimates (table 1). The widely quoted Department of Health estimate of 150 deaths per year (all ages) from serogroup C disease is based on estimates of case fatality ratios from enhanced surveillance of meningococcal disease, ${ }^{2}$ which are similar to our high estimate.

We assumed that in all cases of meningococcal disease patients were admitted to hospital and were recorded in the hospital episode statistics database. We estimated the average length of stay in hospital for specific ages from hospital episode statistics between 1997-8 and 1998-9 and the proportion of cases admitted to an intensive care unit and the length of stay from 1998-9 data (fig 1). We obtained details of the cost of an intensive care bed per day from NHS reference costs (www.doh.gov.uk/nhsexec/refcosts.htm) and the cost of a bed day (non-intensive care) from Unit Costs of Health and Social Care $2000^{9}$ (table 2). There is no national database that documents the use of outpatient services by patients with meningococcal disease, though most patients will require further treatment or follow up. We therefore assumed that every patient attended a first consultation and one follow up appointment at an average total cost of £246 (NHS reference costs, equivalent to $\$ 350$, €403). These costs were varied in the sensitivity analysis between $+/-20 \%$ of the base case.

Survivors of meningococcal disease can have a range of long term sequelae, most commonly hearing impairment, skin scarring, amputation, and neurological disorders. In developed countries 3-15\% of survivors are estimated to have sequelae, ${ }^{10-13}$ with $7 \%$ assumed in the base case. There are likely to be considerable costs associated with the treatment and rehabilitation of survivors with serious sequelae, though there is no published information detailing these costs in the 


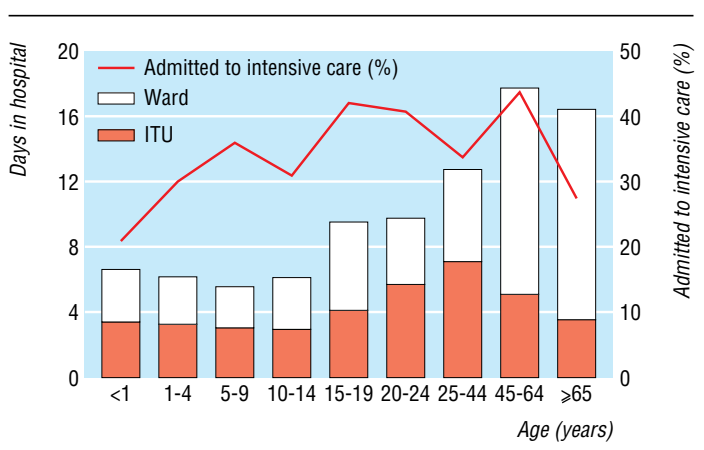

Fig 1 Percentage of cases in which patients were admitted to intensive care unit and mean length of stay in hospital and intensive care, estimated from hospital episode statistics, 1997-8 and 1998-9

United Kingdom. In particular the long term costs to primary care of subsequent permanent chronic disability are difficult to estimate. We assumed that $10 \%$ of survivors with sequelae would require lifetime institutionalised full time care (£21 500 (\$30 637, €35 257) per year). ${ }^{9}$ Survivors with less severe sequelae were assumed to require additional health services at an average cost of $£ 500$ ( $£ 100$ to $£ 1000$ in the sensitivity analysis) per year.

Wider public heath action is recommended after two or more confirmed or probable cases of meningococcal disease probably caused by the same serogroup within an institution. ${ }^{14}$ The primary quantifiable costs are from vaccination with serogroup $\mathrm{C}$ polysaccharide (about $£ 7$ per dose), chemoprophylaxis (about $£ 3$ for $600 \mathrm{mg}$ rifampicin every 12 hours for two days),${ }^{15}$ and swabbing of contacts considered at risk (about £10), with staff costs at about $£ 1$ per procedure. We estimated the average cost of outbreak control per year from 1996 and 1997 data. There were around 11 outbreaks per year of serogroup $\mathrm{C}$ in school and preschool settings (J White, personal communication), with an average of 450 children receiving vaccine and antibiotics and 100 throat swabs taken at an estimated cost of $£ 6500$ per outbreak. We identified further outbreaks in community and university settings in the literature $^{16-20}$ and, when they were not reported, estimated costs according to the numbers vaccinated, given antibiotics, and swabbed. In the base case the estimated cost per year of outbreak control is $£ 245500$. This relates to direct health costs only and not additional external costs incurred (such as admissions due to false alarms, opportunity costs, costs to the schools and universities). Because the number and size of outbreaks cannot be predicted we varied the cost of outbreak control between $+/-20 \%$ in the sensitivity analysis. We assumed that outbreaks would not occur after the start of the vaccination programme, with savings calculated on the basis that the cohort would have been at risk of outbreaks in educational institutions for up to 18 years after the start of the campaign.

\section{Vaccination programme}

The UK vaccine schedule recommends three doses for infants aged under 4 months, two doses for infants aged 5-12 months, and one dose for children and young people aged $1-17$ years..$^{21}$ The $2001 \mathrm{UK}$ list price of the licensed Wyeth meningococcal $\mathrm{C}$ vaccine is
$£ 17.95$ per dose ${ }^{15}$ although bulk purchasing may result in a lower cost. We assumed the vaccine to cost between $£ 8$ and $£ 18$ per dose ( $£ 12$ in the base case). The total cost of the television advertising and leaflet campaign run by the Health Education Authority was $£ 3.5 \mathrm{~m}$ (Health Education Authority, personal communication). By September 2000, 4764 suspected reactions (including headaches, fever, rash, dizziness, faints, seizures) had been reported to the Medicines Control Agency, equivalent to 1:2875 distributed doses, with anaphylactoid reactions reported at a rate of 1 per 500000 doses used. ${ }^{22}$ We assumed that each child with a reported adverse event was seen by a general practitioner at a cost of $£ 18$ per consultation ${ }^{9}$ and that those with anaphylactoid reactions were admitted to hospital at a cost of $£ 310$. ${ }^{9}$

For each child vaccinated at school the Department of Health paid a fee of $£ 1$ to the relevant NHS trust or health authority to cover the cost of nursing, administration, and consumables. General practitioners received item of service payments of $£ 6.25$ per dose of vaccine or $£ 4.30$ for all but the last dose in a series if more than one dose was required. These payments may not accurately reflect the opportunity cost of the campaign. For example, the campaign involved extra administration costs for general practitioners and health authorities, and the normal activities of the school health service (such as health interviews, health promotion) were severely disrupted over the duration of the campaign. To allow for this in the sensitivity analysis we increased the cost of giving any general practice based vaccine to $£ 9$, to reflect the average cost for a consultation with a practice nurse (who is likely to immunise children attending a GP surgery), ${ }^{9}$ and increased school based costs from $£ 1$ to $£ 3$ per dose. In the base case we added an additional $10 \%$ to the vaccine costs to account for wastage, though there may be more wastage in general practice than in schools because of the larger volume of vaccine used in a school setting. We investigated the effects of this in the sensitivity analysis.

Early reports in the United Kingdom suggest an efficacy of $92 \%$ (95\% confidence interval $65 \%$ to $98 \%$ ) for toddlers (aged <2 years) and $97 \%(77 \%$ to $99 \%)$ for teenagers. ${ }^{23}$ In the base case we assumed vaccine efficacy to be $95 \%$ in 2-12 year olds, with toddlers and teenagers as above. We estimated vaccine coverage from routine COVER (cover of vaccination evaluated rapidly) statistics and four one off data collection exercises. On the basis of these data we assumed coverage

Table 2 Unit costs of care and treatment parameters

Parameter

Base value (range)

\begin{tabular}{lc}
\hline Rate of admission to hospital $(\%)$ & $100^{\star}$ \\
\hline Mean cost of intensive care bed/day $(£)$ & $1103(882-1323) \dagger$ \\
\hline Mean cost of paediatric intensive care bed/day $(£)$ & $1196(957-1435) \dagger$ \\
\hline Mean cost of inpatient day (paediatrics) $(£)$ & $310(248-372)^{6}$ \\
\hline Mean cost of inpatient day (general ward) $(£)$ & $223(178-268)^{6}$ \\
\hline Admission to intensive care unit (\%) & $14.9(9.3-23.6, \text { variable by age })^{\star}$ \\
\hline Mean length of stay in intensive care (days) & $3.5(2.5-5.9, \text { variable by age })^{\star}$ \\
\hline Mean length of stay in hospital (days) & $7.9(5.5-17.8, \text { variable by age })^{\star}$ \\
\hline Mean cost outpatient appointment (1st and follow up) $(£)$ & $246(197-295) \dagger$ \\
\hline Cost per general practice consultation $(£)$ & $18(14.40-21.60)^{6}$ \\
\hline Cost per practice nurse consultation $(£)$ & $9(7.20-10.80)^{6}$
\end{tabular}

${ }^{*}$ Hospital episode statistics.

†NHS reference costs. 
Table 3 Units and costs of treating and controlling cases of serogroup C meningococcal disease in 0-17 year olds that arise in one year (base case)

\begin{tabular}{|c|c|c|c|c|}
\hline \multirow[b]{2}{*}{ Parameter } & \multicolumn{2}{|c|}{ Before vaccine } & \multicolumn{2}{|c|}{ After vaccine } \\
\hline & Units & Cost (£) & Units & Cost $(£)$ \\
\hline Cases & 1137 & & 248 & \\
\hline Deaths & 85 & & 21 & \\
\hline Inpatient stay in intensive care & 1127 bed days & 1347900 & 263 bed days & 282300 \\
\hline Inpatient stay on ward & 6522 bed days & 2021900 & 1486 bed days & 460700 \\
\hline Outpatient follow up & 2104 appointments & 258800 & 496 appointments & 61000 \\
\hline Outbreak costs & 11 outbreaks per year & 245550 & 0 outbreaks & 0 \\
\hline Total costs of treating acute meningococcal disease & & 3874250 & & 804000 \\
\hline Long term costs of treating sequelae ${ }^{*}$ & 66 mild & 983000 & 14 mild & 211500 \\
\hline & 7 severe & 4708500 & 2 severe & 1010500 \\
\hline Total costs of treating all cases that arise in one year & & 9565750 & & 2026000 \\
\hline
\end{tabular}

*Present value of cost of treating cases that arise in one year for the rest of their lifetime, discounted at $3 \%$.

to be $89 \%$ in under 1 year olds, $82 \%$ in $1-4$ year olds, ${ }^{24}$ $87 \%$ in $5-13$ year olds, $83 \%$ in $14-15$ year olds, and $65 \%$ in 16-17 year olds in full time education (Marie Rush, personal communication). Limited information is available on vaccination in 16-17 year olds not in education, and we estimated coverage to be $50 \%$.

\section{Results}

In the late 1990s the burden of serogroup C meningococcal disease was considerable, with an estimated 1519 cases in 1998-9, of which 1137 occurred in people aged $0-17$ years. There were 72 deaths due to laboratory confirmed serogroup $\mathrm{C}$ disease in this age group. After adjustment for underascertainment this figure could be as high as 107 deaths. In the absence of a meningococcal $\mathrm{C}$ vaccination programme, the annual costs of treating and controlling acute serogroup $\mathrm{C}$ disease in $0-17$ year olds is estimated to be around $£ 3.87 \mathrm{~m}$. Inclusion of the costs of treating long term sequelae increases this to $£ 9.6 \mathrm{~m}$ (table 3 ).

The vaccination programme should substantially reduce the future burden of disease and associated costs (table 4). The campaign is estimated to prevent 7880 cases and 845 deaths, resulting in nearly 23000 discounted life years saved over the lifetime of the vaccine campaign cohort, given base case assumptions (table 4). This is estimated to avoid costs of about $£ 29 \mathrm{~m}$ present value (base case) in treatment and control. The total cost of vaccinating the campaign cohort was estimated at $£ 172 \mathrm{~m}$ at $£ 12 /$ dose (base case), ranging from $£ 126 \mathrm{~m}$ at $£ 8$ a dose to $£ 241 \mathrm{~m}$ at $£ 18$ a dose.

We estimated the cost per life year saved of the entire programme to be $£ 6259$. The school based cam- paign is more cost effective than the general practitioner based campaign, primarily because of the lower delivery costs per person. Although early vaccination maximises life years saved, routine vaccination at 2, 3, and 4 months is the least cost effective because the cost of giving three doses is substantially higher. In terms of continued routine vaccination, vaccinating children with one dose at 1 year is more cost effective than vaccinating infants at 2,3 , and 4 months. However, delaying vaccination could result in up to 200 potentially preventable cases of serogroup C meningococcal disease in infants under 1 year.

The sensitivity analyses show that the most striking changes in the cost per life year saved occurred when the assumptions about incidence of disease and case fatality ratios were changed (table 5 , fig 2 ). The results were also sensitive to changes in vaccine cost per dose and vaccine efficacy. The cost per life year saved is fairly insensitive to changes in the parameters with the most uncertainty, such as the cost of treating long term sequelae. The choice of discount rate is critical to the outcome (table 6), with the cost per life year saved increasing as the discount rate increases. Adopting the UK Treasury recommended discount rate reduces the cost per life year saved to less than $£ 4000$.

In the multivariate sensitivity analysis we fixed the discount rate at $3 \%$ and compared different scenarios for risk of disease and mortality (fig 2). In the base case (high incidence, medium case fatality ratio) $95 \%$ of the model simulations resulted in a cost per life year saved of less than $£ 10000$. If we assumed a high case fatality ratio and high incidence then $33 \%$ of results were below $£ 5000$ per life year saved. If the incidence in the cohort is low then cost per life year saved is greatly

Table 4 Cost per life year saved of meningitis C vaccination programme, discounted to present value $(3 \%)$

\begin{tabular}{|c|c|c|c|c|c|c|c|c|c|c|}
\hline $\begin{array}{l}\text { Component of } \\
\text { campaign }\end{array}$ & $\begin{array}{c}\text { Delivery } \\
\text { method (No of } \\
\text { doses) }\end{array}$ & $\begin{array}{c}\text { Population } \\
\text { (millions) }\end{array}$ & $\begin{array}{c}\text { Cases } \\
\text { avoided }\end{array}$ & $\begin{array}{l}\text { Deaths } \\
\text { avoided }\end{array}$ & $\begin{array}{l}\text { Life } \\
\text { years } \\
\text { saved }\end{array}$ & $\begin{array}{l}\text { Cost of vaccine } \\
\text { campaign } \\
(£ \mathrm{~m})^{\star}\end{array}$ & $\begin{array}{l}\text { Total cost } \\
\text { savings }(£ \mathrm{~m})\end{array}$ & $\begin{array}{l}\text { Net cost } \\
(£ \mathrm{~m})\end{array}$ & $\begin{array}{l}\text { Cost per } \\
\text { case } \\
\text { avoided }\end{array}$ & $\begin{array}{c}\text { Cost per } \\
\text { life year } \\
\text { saved }\end{array}$ \\
\hline $0-4$ months & GP (3) & 0.275 & 365 & 30 & 832 & 13.4 & 1.2 & 12.2 & 33326 & 14630 \\
\hline 5-11 months & GP (2) & 0.384 & 512 & 42 & 1165 & 12.8 & 1.7 & 11.1 & 21624 & 9493 \\
\hline $1-4$ years & GP (1) & 2.621 & 2422 & 212 & 5879 & 42.6 & 8.3 & 34.3 & 14138 & 5826 \\
\hline 5-17 years & School (1) & 7.857 & 4432 & 539 & 14354 & 96.7 & 17.3 & 79.4 & 17907 & 5529 \\
\hline $\begin{array}{l}\text { 16-17 year olds not in } \\
\text { education }\end{array}$ & GP (1) & 0.654 & 149 & 22 & 569 & 6.6 & 0.7 & 5.9 & 39341 & 10291 \\
\hline $\begin{array}{l}\text { Overall UK campaign } \\
0-17 \text { years }\end{array}$ & GP and school & 11.791 & 7880 & 845 & 22799 & 172.0 & 29.2 & 142.8 & 18112 & 6259 \\
\hline \multicolumn{11}{|c|}{ Alternative strategy (not implemented) } \\
\hline 12 months & GP (1) & 0.659 & 698 & 55 & 1658 & 10.6 & 2.4 & 8.3 & 11878 & 5003 \\
\hline
\end{tabular}

${ }^{*}$ Cost of Health Education Authority advertising campaign distributed according to population size in each age group. 
increased with $25 \%, 53 \%$, and $75 \%$ of simulations resulting in a cost per life year saved of more than $£ 30000$ for high, medium, and low case fatality ratios respectively.

\section{Discussion}

The meningococcal $\mathrm{C}$ vaccination campaign has rapidly and substantially reduced the incidence of serogroup $\mathrm{C}$ meningococcal disease in the targeted age groups. ${ }^{23}$ Modelling of the cost effectiveness of the campaign supports the introduction of the vaccine.

The sensitivity analyses show that assumptions on the incidence of disease are critical in determining the cost effectiveness of the campaign. This incidence cannot be predicted, especially given the variation in and instability of prevalent meningococcal strains. However, disease surveillance since the start of the campaign indicates that incidence of serogroup C disease has continued to increase in people aged 20-25 years, suggesting that in the absence of vaccination the incidence of the disease may have also continued to increase in those aged 0-17 years. This would have resulted in the vaccine campaign being more cost effective than we have estimated.

The current schedule of routine infant immunisation at 2, 3, and 4 months is the most clinically effective but least cost effective strategy because of the higher delivery costs and the three dose schedule. Routine immunisation at 12 months would be more cost effective but could result in up to 200 preventable cases in infants each year. The catch up campaign was most cost effective when delivery was school based rather than general practice based. School and general practice components complemented each other because extra general practice sessions were used to "mop up" children who missed sessions at school to improve coverage.

This analysis ignores gains in quality of life, principally because of a lack of information. Furthermore, we did not incorporate effects of herd immunity into the model because of uncertainty over the transmission dynamics of Neisseria meningitidis. The experience with Haemophilus influenzae type b (Hib) conjugate vaccination in the United Kingdom ${ }^{25}$ (and elsewhere) suggests that conjugate vaccination reduces carriage. If menin-

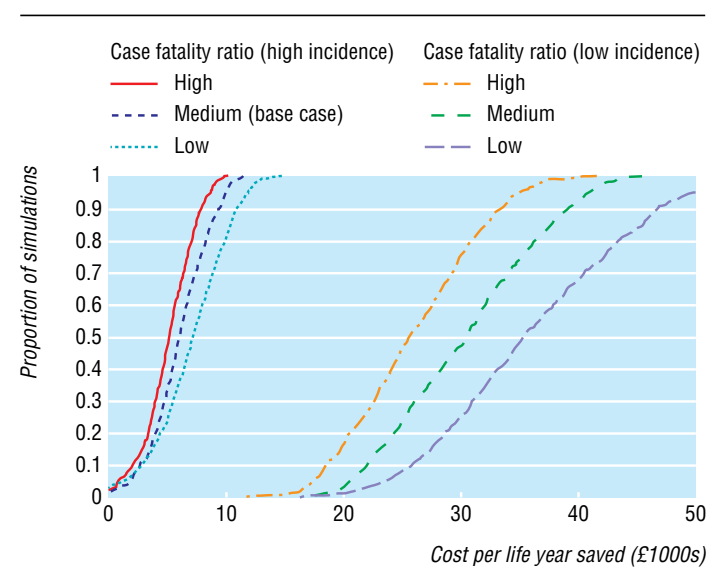

Fig 2 Cost per life year saved estimated from multivariate sensitivity analysis
Table 5 Cost per life year saved of meningococcal $C$ vaccination campaign in 0-17 year olds, results from univariate sensitivity analysis

\begin{tabular}{|c|c|c|}
\hline Parameter (base case ${ }^{\star}$ ) & Range & Cost per life year saved \\
\hline \multirow[t]{5}{*}{ Incidence/case fatality ratio (high/medium) } & High/high & $£ 5295$ \\
\hline & High/low & $£ 7404$ \\
\hline & Low/high & $£ 21699$ \\
\hline & Low/medium & $£ 25459$ \\
\hline & Low/low & $£ 30169$ \\
\hline Vaccine cost per dose (£12) & $£ 8-£ 18$ & $£ 4227-9309$ \\
\hline \multicolumn{3}{|l|}{ Vaccine efficacy: } \\
\hline Toddlers $(92 \%)$ & $65-98 \%$ & $£ 6212-6480$ \\
\hline Children $(95 \%)$ & $71-98 \%$ & $£ 6101-7827$ \\
\hline Teenagers $(97 \%)$ & $77-99 \%$ & $£ 6228-6593$ \\
\hline \multirow[t]{3}{*}{ Wastage $(10 \%)$} & $0 \%$ & $£ 5705$ \\
\hline & $10 \% \mathrm{GP} / 5 \%$ school & $£ 6085$ \\
\hline & $15 \% / 15 \%$ & $£ 6537$ \\
\hline \multicolumn{3}{|l|}{ Delivery costs: } \\
\hline GP $(£ 4.30 / £ 6.25)$ & $£ 9$ & $£ 6800$ \\
\hline School (31) & $£ 3$ & $£ 6842$ \\
\hline$\%$ of survivors with sequelae $(7 \%)$ & $3-15 \%$ & $£ 5850-6353$ \\
\hline \multicolumn{3}{|l|}{ Cost of sequelae: } \\
\hline Severe (£21 500/year) & $£ 10000-50000$ & $£ 6134-6310$ \\
\hline Mild (£500/year) & $£ 100-1000$ & $£ 6240-6275$ \\
\hline Hospital costs (see table 2) & $\pm 20 \%$ & $£ 6055-6464$ \\
\hline Cost outbreaks/year (£245) 500 & $\pm 20 \%$ & $£ 6230-6289$ \\
\hline
\end{tabular}

*Under base case scenario cost per life saved is $£ 6259$.

Table 6 Cost per life year saved of meningitis $C$ vaccine programme in 0-17 year olds, with varying discount rates, base case parameter estimates otherwise

\begin{tabular}{lcc} 
Benefits & Costs & Cost per life year saved \\
\hline $0 \%$ & $0 \%$ & $£ 1655$ \\
\hline $1.5 \%$ & $1.5 \%$ & $£ 3438$ \\
\hline $3.0 \%$ & $3.0 \%$ & $£ 6259$ \\
\hline $4.0 \%$ & $4.0 \%$ & $£ 8815$ \\
\hline $5.0 \%$ & $5.0 \%$ & $£ 11957$ \\
\hline $6.0 \%$ & $6.0 \%$ & $£ 15710$ \\
\hline $1.5 \%$ & $6.0 \%$ & $£ 3845$ \\
\hline
\end{tabular}

gococcal $\mathrm{C}$ vaccination reduces transmission of serogroup $\mathrm{C}$ meningococci, the risk of infection for those who have not been vaccinated would decline. The net effect of these omissions would be that the campaign was probably more cost effective than is presented here.

We thank Joanne White, Marie Rush, Usha Gungabissoon, Katy Davison, and Mary Ramsay from the Immunisation Division, Communicable Disease Surveillance Centre, Colindale, and Ed Kaczmarski (Public Health Laboratory Service, Meningococcal

Reference Unit, Manchester) for providing information and assistance.

Contributors: Both authors designed, conducted, analysed, and wrote up the study. WJE is guarantor.

Funding: WJE is funded by the MRC (grant No G9818303). Competing interests: None declared.

1 Chief Medical Officer, Chief Nursing Officer, and Chief Pharmacist. Introduction of immunisation against group C meningococcal infection. London: Department of Health, 1999 (PL/CMO/99/02).

2 Miller E, Salisbury DM, Ramsay ME. Planning, registration, and implementation of an immunisation campaign against meningococcal serogroup C disease in the UK: a success story. Vaccine 2001;20:S58-67.

3 Richmond P, Borrow R, Miller E, Clark S, Sadler F, Fox A, et al. Meningococcal serogroup $\mathrm{C}$ conjugate vaccine is immunogenic in infancy and primes for memory. J Infect Dis 1999;179:1569-72.

4 Begg N. Outbreak management. In: Cartwright K, ed. Meningococcal disease. Chichester: Wiley, 1995:285-305.

5 Gold MR, Siegel JE, Russell LB, Weinstein MC. Cost-effectiveness in health and medicine. Oxford: Oxford University Press, 1996.

6 Department of Health. Policy appraisal and health.London: Department of Health, 1995.

7 Ramsay M, Collins M, Rush M, Kaczmarski E. The epidemiology of meningococcal disease in England and Wales, 1996 and 1997. Eurosurveillance 1997;2:1-2 


\section{What is already known on this topic}

The burden of group $\mathrm{C}$ meningococcal disease in England and Wales in the late 1990s was considerable

In November 1999 the United Kingdom was the first country to introduce mass vaccination against group C meningococcal disease

There are no published economic evaluations of the vaccination campaign

\section{What this study adds}

This economic evaluation supports the introduction of the meningococcal $\mathrm{C}$ vaccine

School based vaccination is more cost effective than routine vaccination of infants because delivery costs are lower and fewer doses are required

8 Wylie PA, Stevens D, Drake W 3rd, Stuart J, Cartwright K. Epidemiology and clinical management of meningococcal disease in west Gloucestershire: retrospective, population based study. BMJ 1997;315:774-9.

9 Netten A, Curtis L. Unit costs of health and social care 2000. Canterbury: Personal Social Services Research Unit, University of Kent, 2000.

10 Baraff LJ, Lee SI, Schriger DL. Outcomes of bacterial meningitis in children: a meta-analysis. Pediatric Infect Dis J 1993;12:389-94.

11 Erickson L, De Wals P. Complications and sequelae of meningococcal disease in Quebec, Canada 1990-1994. Clin Infect Dis 1998;26:1159-64.

12 Barquet N, Domingo P, Cayla JA, Gonzalez J, Rodrigo C, FernandezVildarich P, et al. Meningococcal disease in a large urban population (Barcelona, 1987-1992). Arch Intern Med 1999;159:2329-40.
13 Schildkamp RL, Lodder MC, Bijlmer HA, Dankert J, Scholten RJ. Clinical manifestations and course of meningococcal disease in 562 patients. Scand J Infect Dis 1996;28:47-51.

14 Stuart JM, Monk PN, Lewis DA, Constantine C, Kaczmarski EB, Cartwright KA. Management of clusters of meningococcal disease. Commun Dis Rep CDR Rev 1997; 7:R3-5.

15 British Medical Association, Royal Pharmaceutical Society of Great Britain. British National Formulary 39. London: BMA, Royal Pharmaceutical Society of Great Britain, 2000.

16 Irwin DJ, Miller JM, Milner PC, Patterson T, Richards RG, Williams DA, et al. Community immunization programme in response to an outbreak of invasive Neisseria meningitidis serogroup $\mathrm{C}$ infection in the Trent region of England 1995-1996. J Public Health Med 1997;19:162-70.

17 Barker RM, Shakespeare RM, Mortimore AJ, Allen NA, Solomon CL, Stuart JM. Practical guidelines for responding to an outbreak of meningococcal disease among university students based on experience in Southampton. Commun Dis Public Health 1999;2:168-73.

18 CDSC. Meningococcal disease in Ironville. Commun Dis Rep CDR Wkly 1999;9:297

19 CDSC. Outbreak of meningococcal disease in students in Cardiff. Commun Dis Rep CDR Wkly 1996;6:311.

20 CDSC. Clusters of meningococcal disease in university students. Commun Dis Rep CDR Wkly 1997;7:393-6.

21 Anon. Meningococcal disease [replacement chapter]. In: Salisbury DM, Begg NT, eds. Immunisation against infectious disease. London: HMSO, 1999.

22 Anon. Safety of meningococcal group C conjugate vaccines. Current Problems in Pharmacovigilance 2000;26:14.

23 Ramsay ME, Andrews N, Kaczmarksi EB, Miller E. Efficacy of meningococcal serogroup $\mathrm{C}$ conjugate vaccine in teenagers and toddlers in England. Lancet 2001;357:195-6.

24 CDSC. COVER programme: April to June 2001. Commun Dis Rep Weekly [serial online] 2001;11:immunisation. www.phls.org.uk/publications/ CDR\%20Weekly/archive/immunisationarchive.html (accessed Feb 2002).

25 Barbour ML, Mayon-White RT, Coles C, Crook DW, Moxon ER. The impact of conjugate vaccine on carriage of Haemophilus influenzae type b. J Infect Dis 1995;171:93-8.

(Accepted 8 November 2001) 